\title{
CORRECTION OF SECOND ORDER CHROMATICITY AT TEVATRON *
}

\author{
A. Valishev ${ }^{\dagger}$, G. Annala, V. Lebedev, R.S. Moore, Fermilab, Batavia, IL 60510, USA
}

\section{Abstract}

Correction of the second order betatron tune chromaticity is essential for operation at the working point near half integer resonance which is proposed as one of the ways to improve performance of the Tevatron. In this report the new chromaticity correction scheme with split sextupole families is described. Details of implementation and commissioning at the present working point are discussed.

\section{INTRODUCTION}

The Tevatron Run II luminosity upgrade project envisages the collider operation at the peak luminosity of up to $3 \times 10^{32} \mathrm{~cm}^{-2} \mathrm{~s}^{-1}$. This level of performance may be achieved by increasing the beam intensity or by stronger focusing of the beams at the collision points. The latter opportunity has been exercised in 2005 when the value of beta-function was decreased from $35 \mathrm{~cm}$ to $28 \mathrm{~cm}$ [1]. Further steps in this direction are not feasible because of the relatively large bunch length which can not be easily reduced. Thus, the drop of the hourglass factor caused by the beta-function change diminishes the positive effect on the luminosity.

Increase of the beam intensity leads to the rise of the beam brightness which characterizes the strength of beambeam effects [2]. Currently, the total head-on beam-beam tuneshift $\xi$ for antiprotons reaches 0.025 , for protons its value is up to 0.02 . Since the present betatron tune working point is confined between the $4 / 7$ and $3 / 5$ resonance lines, the available tune space is only 0.028 which makes further growth of $\xi$ virtually impossible.

As a possible way to overcome this limitation a major change of the betatron tune is being considered. Two possible candidates offer an opportunity to open the tune space: the so-called SPS working point near $1 / 3$ resonance, and working point near the half integer. Sextupole nonlinearities in the Tevatron are quite strong thus making the $1 / 3$ resonance a less attractive option.

At the same time, operation near the $1 / 2$ resonance requires careful correction of focusing errors, including the chromatic perturbations. Moreover, simulations predicted that elimination of chromatic dependence of the beta-function at the main IPs may improve proton life time even at the present working point [3]. In the Tevatron, the value of chromatic beta-function beating is directly related to the second-order chromaticity of the betatron tune. Thus we consider that both terms have similar meaning.

\footnotetext{
* Work supported by the Fermi Research Alliance, under contract DEAC02-76CH03000 with the U.S. Dept. of Energy.

†valishev@fnal.gov
}

This report presents the results of design, development and implementation of the new sextupole chromaticity correction at the Tevatron. We describe basic principles used to build the new circuits and compare the calculated chromatic beta-functions with measured parameters at the injection energy and at $980 \mathrm{GeV}$.

\section{BETA-FUNCTION CHROMATICITY}

One can use the well-known perturbation theory approach to describe the distortion of the beta-function caused by chromatic errors (see e.g. [4]).

Horizontal beta-beating excited by a single quadrupole for an off-momentum particle can be described by the formula

$$
\frac{\Delta \beta}{\beta}(s)=-\frac{\delta}{2 \sin (2 \pi Q)} \frac{K}{B \rho} \tilde{\beta} \cos (2|\tilde{\psi}-\psi(s)|-2 \pi Q)
$$

Here $\delta=\Delta p / p$ is the relative momentum deviation, $Q$ is the betatron tune, $K$ is the quadrupole integrated strength, $B \rho$ is the magnetic rigidity, $\psi$ is the betatron phase and $\sim$ denotes values at the location of the quadrupole. In the first order approximation the contributions from all quadrupoles are summed to give the total beta-wave. Quadrupoles of the final focus have the largest strength and highest value of beta-function. In the Tevatron, the betatron phase advance between the two interaction regions is close to $\pi$, thus the contributions from the final focus quadrupoles add with the same phase and they dominate the chromatic $\Delta \beta / \beta$.

The contribution to second-order chromaticity of the horizontal tune derived from the perturbation theory is given by the following expression

$$
\frac{d^{2} Q}{d \delta^{2}}=\left(\frac{1}{4 \pi} K \tilde{\beta}\right)\left(\frac{\Delta \beta}{\beta} / \delta\right)
$$

This effectively means that the second-order chromaticity is proportional to the strength of the final focus quadrupole and to the value of chromatic beta-function at its location.

Figure 1 shows the comparison of the measured and modeled chromatic beta-function of the Tevatron in the collision mode. The measured parameters were obtained from two ORM measurements [1] performed at different values of the revolution frequency. The modeled curve was obtained by the perturbation theory. In Fig. 2 the same betafunctions are plotted on a zoomed horizontal scale close to the CDF interaction region. One can see that the model gives quite accurate description of the effect. 


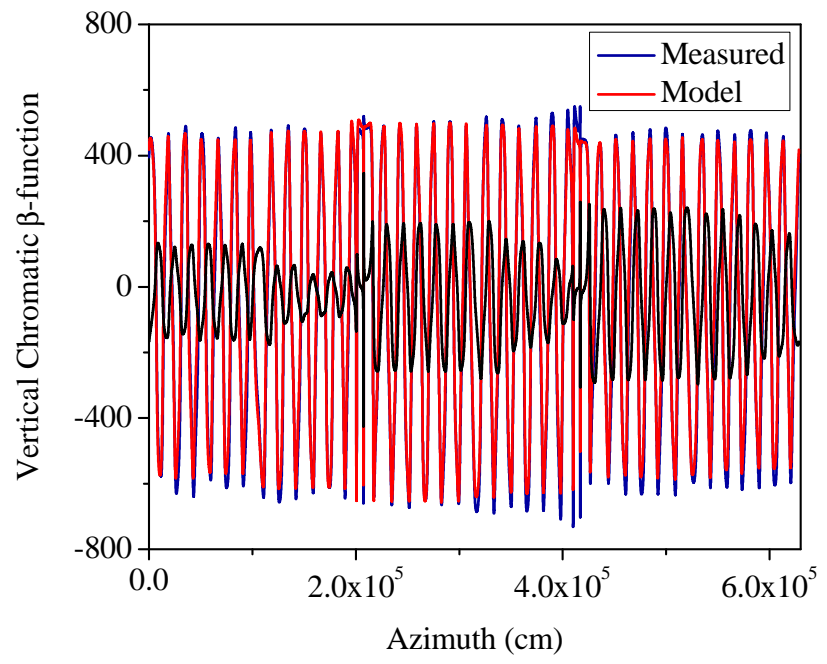

Figure 1: Chromatic beta-function vs. azimuth starting at F0. Blue line - measured, red - model, black - proposed correction.

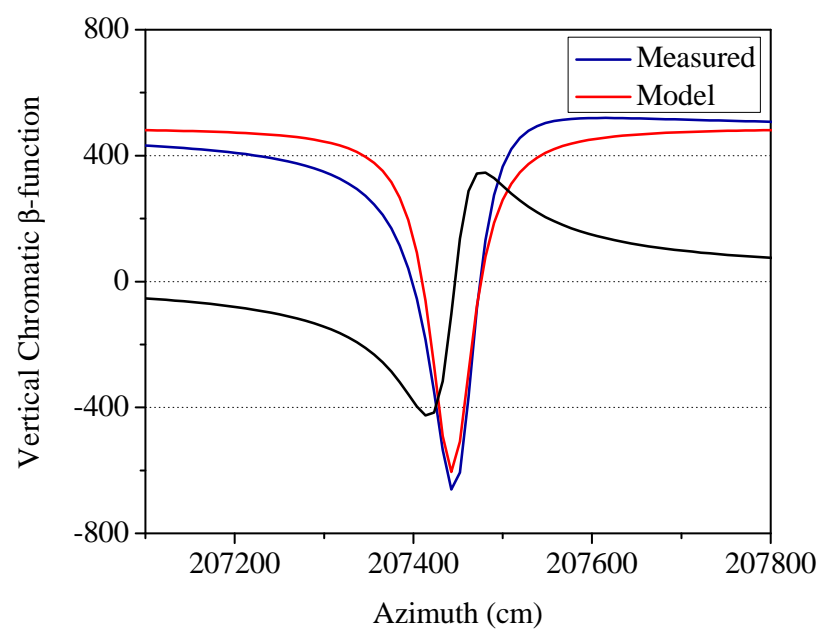

Figure 2: Chromatic beta-function vs. azimuth in the vicinity of CDF IP. Blue line - measured, red - model, black proposed correction.

\section{SEXTUPOLE CORRECTION SCHEME}

Sextupole magnets can be used to correct the secondorder chromaticity generated by quadrupole focusing errors. Using the same perturbative approach and considering sextupole as a quadrupole at the off-center orbit we get the expression for the sextupole-driven horizontal chromatic beta-function:

$$
\frac{\Delta \beta}{\beta}(s)=\frac{\delta}{2 \sin (2 \pi Q)} \frac{S \tilde{D}_{x}}{B \rho} \tilde{\beta} \cos (2|\tilde{\psi}-\psi(s)|-2 \pi Q)
$$

where $S$ is the sextupole strength and denotes parameters at the location of the sextupole. Even though the effect of individual sextupoles on the beta-function chromaticity is much less than that of the final focus quadrupoles, one can achieve compensation by selecting many sextupoles with the correct phase advance and combining them into families.

There are 176 chromaticity correction sextupole magnets in the Tevatron. Originally, they were combined into two families SF and SD, each with 88 elements powered in series. Sextupole coils are placed in the so-called spool pieces located next to quadrupoles in the regular FODO lattice of the arcs. The betatron phase advance per FODO cell is close to 60 degrees in both planes. Thus, it was possible to select sextupoles that would have their betatron phase advance with respect to the final focus quads equal to $\pi$ or $\pi / 2$. The total of 46 sextupoles in each family were found to satisfy this condition. However, it was discovered that rewiring them into 4 new circuits would have considerable cost mainly due to the large amount of required new cable. Hence, we had to limit the number of elements in the new circuits and group them close to three service buildings which would reduce the length of the new cabling. The final configuration is shown in Fig. 6. The total of 44 sextupoles were taken out of the SF and SD families (22 from each). The sextupoles are powered by 12 new power supplies and logically grouped into 4 families. The new circuits are designed to have equal number of elements with positive and negative current. This allows to keep the linear betatron tune chromaticity constant when using the new groups. The disadvantage of this solution is that it breaks the 6-fold symmetry of the machine but the expected feeddown effect on the beta-functions is small.

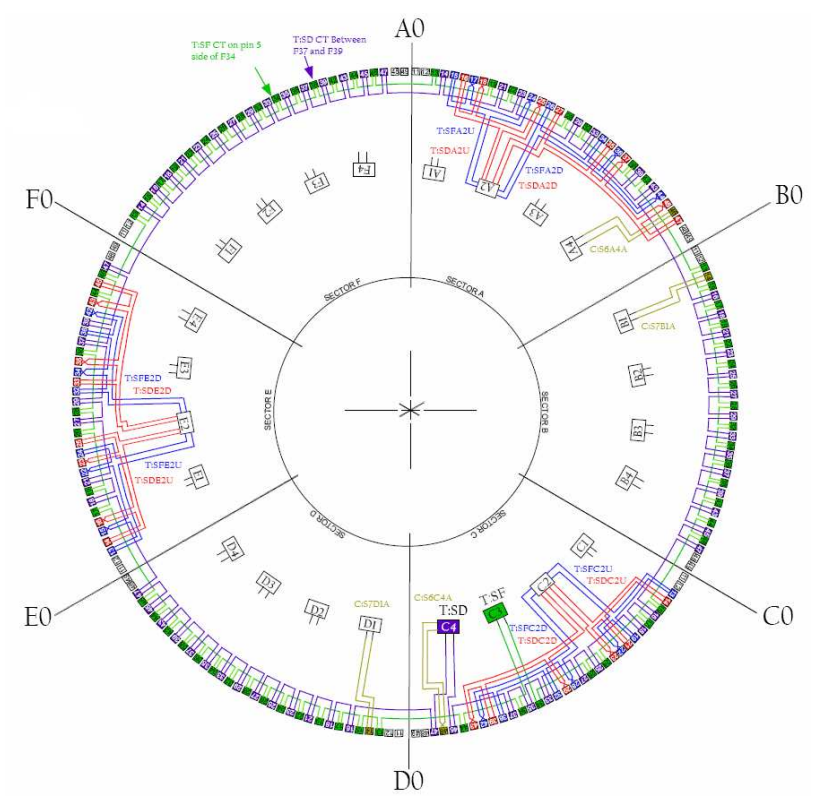

Figure 3: Layout of sextupoles in the Tevatron.

In Figures 1,2 and 4 the simulated effect of application of the new families on the chromatic beta-function is plotted for the collision and injection modes. Note that the expected beta-function chromaticity at the CDF IP at collisions is close to zero. 


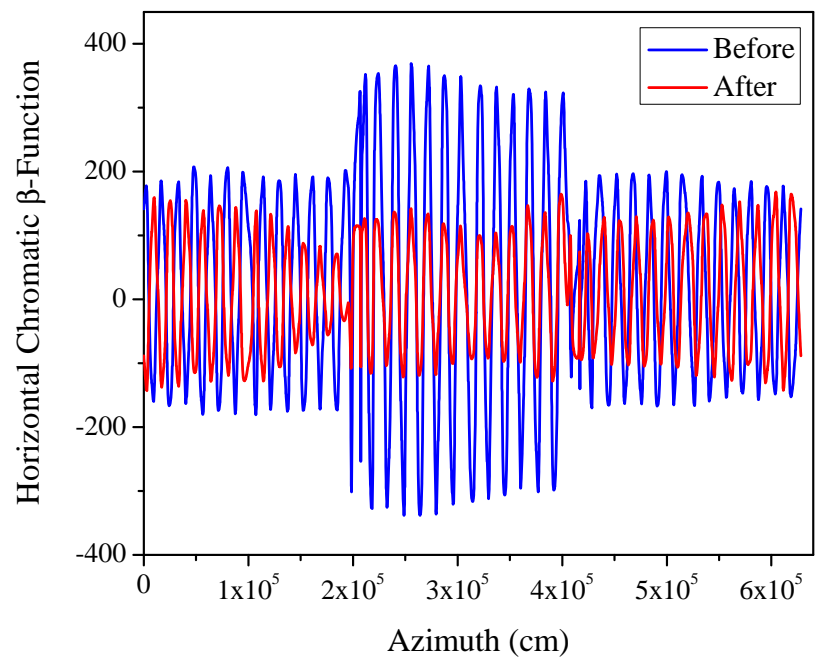

Figure 4: Horizontal chromatic beta-function at the injection energy. Blue line is for the original sextupole configuration, red - for the proposed correction.

\section{RESULTS}

The new sextupole scheme was commissioned in three stages. First, the chosen elements were switched to the new power supplies, while being operated at the original current and polarity. This allowed to verify the stability and reliability of electrical components. Second, the new circuits were turned off and the Tevatron was tuned for operating in this new mode. Finally, polarities of some of the sextupoles were flipped and the new families were turned on at the designed strength.

Figures 5 and 6 show the comparison of expected and measured beta-function chromaticity after implementation of the new circuits in the injection and collision modes, respectively. The corresponding change in the second-order tune chromaticity is presented in Fig. 7.

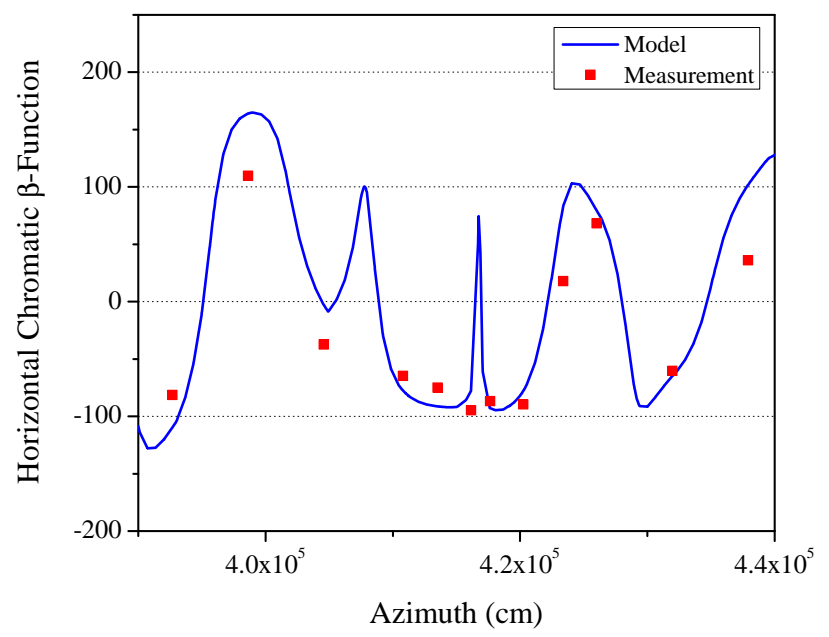

Figure 5: Chromatic beta-function near the D0 IP at the injection energy.

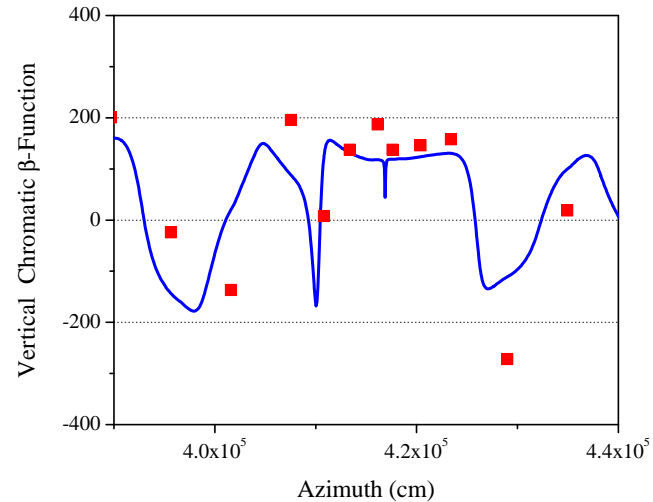

Figure 6: Chromatic beta-function near the D0 IP in the collider mode.

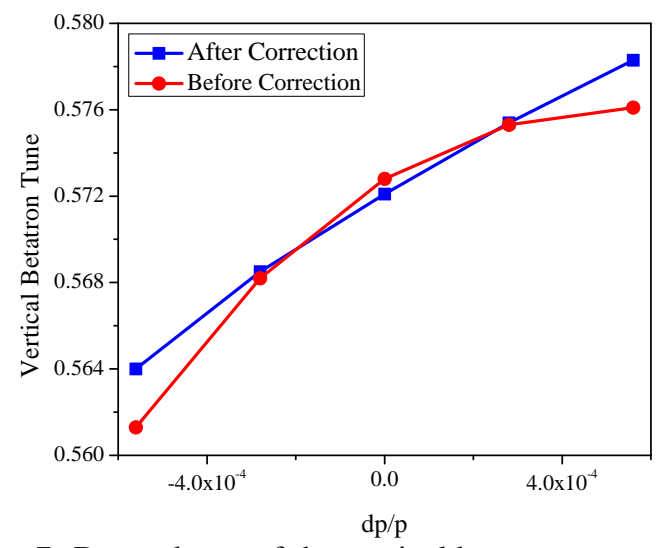

Figure 7: Dependence of the vertical betatron tune on particle momentum in the collider mode.

Measured beta-functions are in good agreement with the calculated values. The second-order tune chromaticity in the collision mode was reduced from -15000 units to -3000 units which is close to expected. As the result, we observe a noticeable improvement of the proton beam lifetime at collisions [3]. Commissioning of the new circuits allows to consider the new working point near the half integer resonance.

\section{ACKNOWLEDGMENTS}

The authors wish to thank D. Still, B. Hanna, D. Bollinger and many others in the Tevatron group for their help in implementation of the new circuits in operations.

\section{REFERENCES}

[1] A. Valishev et al., "Progress with Collision Optics of the Fermilab Tevatron Collider", EPAC2006, Edinburgh, Scotland, 2006

[2] V. Shiltsev et al., Phys. Rev. ST AB, 8101001 (2005)

[3] A. Valishev et al., "Observations and Modeling of BeamBeam Effects at the Tevatron Collider", these Proceedings

[4] A.W. Chao, M. Tigner, "Handbook of Accelerator Physics and Engineering", World Scientific, Singapore, 1999, pp. 263-264 